Abstract ID: 39

\title{
The mid term functional outcomes of NexGen legacy posterior stabilized flex fixed bearing total knee replacement in primary osteoarthritis
}

Ed Simor Khan Mor Japar Khan ${ }^{\mathrm{a}}$ | Rizal Abdul Rani ${ }^{\mathrm{b}}$ | Mohd Ashraff Mohd Ariff $^{\mathrm{b}}$ | Khairul Nizam Siron@Baharom ${ }^{\mathrm{a}}$ | Mohd Shahidan Noor Rahin ${ }^{\mathrm{a}}$ | Nor Hamdan Mohd Yahaya ${ }^{\mathrm{b}}$

${ }^{a}$ Kulliyyah of Medicine, International Islamic University Malaysia

${ }^{b}$ Universiti Kebangsaan Malaysia Medical Centre

Introduction: To evaluate the midterm functional outcomes of patients who had undergone total knee replacement (TKR) using posterior stabilized (PS) fixed bearing system from January 2005 until April 2006 for primary osteoarthritis of the knees. Methods: A retrospective study was done on forty-six patients, $87 \%$ female $(n=40)$ and $13 \%$ male $(n=6)$, aged 54 to 81 years old (mean $69 \pm 5.69$ ) who had undergone primary TKR using the NexGen LPS-Flex Zimmer Warsaw. The patients were followed up for at least five years. A medial parapatellar approach and cemented posterior stabilized fixed bearing TKR system were used performed by one of two arthroplasty surgeons. All patients followed the same rehabilitation protocol post-operatively. Assessments were done using Oxford-12 knee scoring questionnaire to evaluate knee function and SF-36 to assess quality of life outcomes. Results: Oxford knee score revealed patients had lower (better) than average scores in terms of function (score $14.35 \pm 4.21$ out of 35 ) and pain (score $9.28 \pm 3.01$ out of 25 ). Analysis on the quality of life outcome score with SF-36 revealed a higher (better) score for mental component summary (MCS) relative to physical component summary (PCS). Patients had the highest score for role emotional (RE), followed by mental health $(\mathrm{MH})$, and social functioning (SF). Conclusions: The NexGen LPS-Flex TKR produced a good functional outcome with minimal pain in the operated knee with better mental compared to physical health of patients in the midterm period post-total knee replacement.

KEYWORDS: Total knee replacement, midterm functional outcome 